\title{
Hairy Concerns: Use of Instructional/Informational Sheets for Assistance with Disorders of Hair
}

\author{
Craig G. Burkhart ${ }^{*}, 1$ and Craig N. Burkhart ${ }^{2}$ \\ ${ }^{I}$ University of Toledo College of Medicine, USA; Ohio University College of Osteopathic Medicine, 5600 Monroe Street, \\ Suite 106B, Sylvania, OH 43560, USA \\ ${ }^{2}$ Department of Dermatology, Northwestern University, Chicago, Illinois, USA
}

\begin{abstract}
Hair serves as a social sign of gender, age, status, values, and group membership. Hair is a major part of one's self-identity, and central to one's feeling of personal attractiveness. Treating a patient with hair loss and/or hair issues is not easy because of psychosocial overtones and patients' distress and body image concerns. In this paper, several common hair diseases will be discussed in consort with sample patient instructional sheets. They include trichorrhexis nodosa, telogen effluvium, pattern alopecia, alopecia areata, excess facial hair in females, and male pattern baldness. This paper therefore presents a template from which individual physicians can assess whether any portion of the material is worth being incorporated into their individual practices.
\end{abstract}

Scalp hair has been endowed with greater social and psychological significance than biological importance. Medically, it merely provides some shielding from ultraviolet rays and some cushioning of the cranium from head gear. Historically and culturally, hair serves as a social sign of gender, age, status, values, and group membership. As stated previously, "from monks to skinheads, prisoners of war to warriors, bigwig European aristocrats to mop top Beatles, and hippies to head-shaven celebrity athletes, hair makes a statement whether chosen or imposed [1]".

Hair is a major part of one's self-identity, and central to one's feeling of personal attractiveness. Physical appearance symbolizes one's perceptions, thoughts, feelings, and behaviors. People use this physical attribute to express one's individuality. Hair styling is a major part of one's daily grooming ritual. Quoting a psychologist, "Preparing one's hair is preparation to face one's social world. The expression 'bad hair day' is testimony to the psychological importance of hair. Hair loss can turn every day into a bad hair day [1]".

Treating a patient with hair loss and/or hair issues is not easy because of psychosocial overtones and patients' distress and body image concerns. These patients often require considerable support. They need education on their condition as well as a realistic appraisal of possible treatments, length of time before resolution, and alternatives in terms of hair replacement. After discussing the 'hairy' situation, patients who remain greatly distressed and/or are totally unrealistic with expectations of magical cures, one might ask them to go to the internet or book store and research their stated hair disease as well as the topic of 'body image.' In short, for some individuals, the loss of hair represents a body image impairment which can undermine self-esteem and quality of life.

\footnotetext{
*Address correspondence to this author at the University of Toledo College of Medicine, 5600 Monroe Street, Suite 106B, Sylvania, OH 43560, USA; Tel: 419-885-3403; Fax: 419-885-3401;

E-mail: cgbakb@aol.com
}

In this paper, several common hair diseases will be discussed in consort with sample patient instructional sheets. This paper therefore presents a template from which individual physicians can assess whether any portion of the material is worth being incorporated into their individual practices. One option is to allow patients time to read the informational sheet after completing the history and physical, and therefore, before outlining the treatment program.

\section{TRICHORRHEXIS NODOSA}

Unlike most physical characteristics, hair can be altered and manipulated to the dictates of culture and fashion. The chemical reactions from coloring, perming, and straightening hair affect the normal structure of the hair shaft. Many hair cosmetics are used without any visual effects on the integrity of hair, they nonetheless cause microscopic weathering rendering the hair structure weaker and more prone to breakage. When hair breakage occurs with visible hair loss, the term that is utilized is 'trichorrhexis nodosa [2]'.

The trichorrhexis nodosa sheet (informational sheet 1) is very informative as patients with this entity often question the validity of this disease entity. Hair styling and hair cosmetic habits are hard to change, but after reading through this synopsis, my patients are more receptive to considering altering their hair style and using fewer chemicals.

\section{TELOGEN EFFLUVIUM}

When presenting telogen effluvium (informational sheet 2) to patients, one has to familiarize patients with the concept that our hair grows in cycles. At any given time, a small percentage of our hair follicles are in the stage in which hair is shed in preparation for new hair that follows. One loses hair every day. Evidence for shedding is noted in one's hair brush as part of one's normal grooming routine. With telogen effluvium, there is a shift in the growth cycle in which an abnormal number of follicles have moved into the telogen, or resting, phase of the growth cycle. 
Nutritional factors are mentioned on the informational sheet for this condition. Rather than elaborate studies, our minimal knowledge on the subject comes from studies of protein-energy malnutrition, starvation, and eating disorders [3]. Otherwise healthy individuals may not find great benefit in dietary supplementation. However, there is medical evidence that major deficiencies in zinc and biotin can cause hair loss, so it doesn't hurt to offer patients these inexpensive agents that may be somewhat helpful. In the very least, it allows more time for the body to return to normal hair cycling and reduce the amount of hair shedding. To a great extent, time heals telogen effluvium, especially if the stresses that caused the hair shedding are eliminated.

\section{PATTERN ALOPECIA IN WOMEN}

Pattern alopecia is the result of genetics, aging, and hormonal changes that combine to cause changes in the hair follicle, namely, miniaturization of the terminal hair into vellus hair. The end result is generalized thinning of hair over the crown and top areas of the head. The patient handout (informational sheet 3 ) divides the condition into pattern alopecia secondary to low estrogen levels and pattern alopecia secondary to an increase in (what is often referred to as) male hormones (specifically testosterone and 5dihydroxyepiandrosterone). The sheet further outlines the options of treatment for patients experiencing low estrogen.

\section{ALOPECIA AREATA}

Alopecia areata (informational sheet 4) is a non-scarring hair loss condition which can affect any hair-bearing surface. The only predictable thing about the progress of the condition is that it is unpredicatable. Several episodes of hair loss and hair regrowth are not unusual in this condition. It is reported that as many as $7 \%$ of individuals with alopecia areata develop permanent hair loss in patches or in significant portions of their scalp. Alopecia areata is due to a hair cycle dysfunction, but the pathogenesis of the condition is still unknown, with non-specific immune factors appearing to be contributory.

At present, there is no cure that works every time. Because of the chronic nature of the disease, any mode of treatment needs to be used for prolonged periods of time. The prognosis tends to be worse when the condition begins in early childhood and when extensive areas of the scalp as involved. Thus, patients devoid of scalp hair (alopecia totalis) and/or total body hair (alopecia universalis) normally have a poor prognosis.

Having tried innumerable treatments through the years, I presently start with topical immunomodulators and a keratolytic agent [4]. The treatment can be performed by patient at home and response rates have been very encouraging. Indeed, patients are asked to call for reappointments in 2 to 3 months if no growth is experienced.

For non-responders, I consider intralesional corticosteroid injections for localized patchy alopecia areata. Normally a concentration of $2.5 \mathrm{mg} / \mathrm{mL}$ is used and a total of $3 \mathrm{~mL}$ of triamcinolone acetonide would be the most utilized in any one visit. A 30 gauge needle is helpful for the intradermal injections. In areas of total hair loss, a series of blebs are raised with approximately $0.1 \mathrm{~mL}$ per site, $1 \mathrm{~cm}$ apart. Such a treatment would be repeated every 6 weeks, if proving successful.

The other options which I consider in recalcitrant cases includes sulfasalazine $[5,6]$. This is an anti-inflammatory drug composed of sulfonamide and a salicylate which has shown to be of benefit for rheumatoid arthritis, arthritis, inflammatory bowel disease, psoriasis, and dermatitis herpetiformis. It has numerous immunological effects including immunosuppression and immunomodulation. It leads to inhibition inflammatory cell chemotaxis, cytokine production, antibody proliferation, and IL-2 release. Other alternatives for treatment include topical glucocorticoids, anthralin (Dithranol, Drithocreme), and topical minoxidil.

Topical immunotherapy has been recommended for extensive alopecia areata that fails with conventional therapy [7]. The three contact sensitizers that have been used are dinitrochlorobenzene (DNCB), squaric acid dibutyl ester (SADBE), and diphenylcyclopropenone (DPCP). DNCB has shown mutagenic effects and is rarely used presently. The contact sensitizer is applied weekly trying to put a concentration of the sensitizer to maintain a low-grade tolerable erythema, scaling, and pruritus on the treated side for 36 hours after application. Side effects of such treatment included eczema, possible extention of the dermatitis to other body parts, itching, edema, lymphadenopathy, and pigmentary alterations.

\section{EXCESS FACIAL HAIR IN A WOMAN}

While hair loss is devastating to women, an equally troubling problem is unwanted hair growth. Our society has deemed body hair on women is undesirable, and women have developed a habit of shaving such unwanted hairs as on the underarms and legs. Indeed, the sight of a woman with natural hair development that is unshaven is considered grotesque. Thus, this is another example of multimillion dollar industries motivating women to try to alter their bodies to match some ideal seen in fashion magazines. Thus shaving joins dieting, exercise, cosmetics, hair dying, and cosmetic surgeries as the method to achieve beauty in our society. These body-altering behaviors certainly attest to the fact that a woman's body is not acceptable the way it naturally is [8].

Millions of women experience what could be considered heavy hair growth on the face. Some ethnic background and genetic parentage normally have some facial hair, which in our generation, is still considered undesirable. Additionally, abnormal hair growth can also accompany altered androgen metabolism, increase androgen production, decreased androgen binding in the circulation, and/or exogenous androgens.

An overview of available methods for hair removal is discussed on the instructional sheet for excess facial hair (informational sheet 5). There is hope that safer, more selective, and more economical lasers will be developed for hair removal.

\section{MALE PATTERN BALDNESS}

Male pattern baldness is a genetically predisposed condition mediated by androgen metabolism. It is not a disease in the conventional sense, but in our culture, hair loss can threaten one's sense of personal and social acceptance and self-esteem. 
There has been significant advance in the medical treatment of androgenetic alopecia with two very good treatment options. Finasteride is a type II 5-alpha-reductase inhibitor, and thereby blocks the major enzyme responsible for the condition. The drug has been shown to slow the progression of hair loss and leads to some regrowth of hair recently lost. Although studied most extensively in males under the age of 50 , it also has benefit for those in the sixth and seventh decade of their life as well. Additionally, the side effect of reducing prostatic size is more welcomed with these latter age group. I prefer giving the flier provided by the manufacturer for finasteride, rather than an informational sheet, because it contains a monetary rebate and adequate information on the drug.

The other product for male pattern alopecia is minoxidil. The method of action of minoxidil is not as direct as finasteride. Nevertheless, clinical results are satisfactory with minoxidil. There is some evidence with monkeys and with several human case reports, that maximum benefit is achieved when both finasteride orally and minoxidil topically are administered to balding individuals; but given monetary constraints, if one is choosing one product, I would vote for finasteride.

Presently the preferred surgical approach to male pattern baldness is hair micrografts. With this method, tiny follicular units of one to four hairs are inserted in closely spaced fashion over the entirety of the bald or thinning areas. In our practices, such a procedure would be referred to a different physician.

\section{ADDITIONAL INFORMATION}

\section{General Information on Trichorrhexis Nodosum}

Trichorrhexis nodosum is a distinctive type of hair loss resulting from physical and/or chemical trauma to the hair shaft. It clinically presents with patchy or diffuse hair loss. The hair readily fractures and snaps off on mild pulling or combing, and the hair is not able to achieve its normal length. The base of the hair still is present, and will proceed to grow out if the offending physical and chemical agents are discontinued, or greatly diminished.

The most common cause of trichorrhexis nodosum is hair straighteners which are also referred to as hair relaxers. There are two types of hair straighteners, namely lye relaxers containing sodium hydroxide as the active ingredient, and "no-lye" products in which calcium hydroxide and guanidine carbonate are mixed to form guanidine hydroxide. Both types of relaxers can cause trichorrhexis nodosum, although "no-lye" products tend to have less incidence of scalp irritation.

With both straightening products, one needs to follow common safety rules in terms of leaving the products in for no longer than the prescribed time, carefully washing out with neutralizing shampoo, and following up with regular conditioning. Conditioners are very important as they restore some of the natural oils and proteins removed by these chemicals. Blow drying and curling further damage the hair after relaxers and should be avoided. Also, coloring the hair after using a relaxer also increases the risk of hair damage. The FDA actually recommends the use of only semi-permanent hair dyes to relaxed hair as it has no ammonia and less peroxide than permanent color. Moreover, it is standard to wait at least two to four weeks before applying any hair color to recently relaxed hair.

The most common misuse of relaxers is overprocessing. Reapplications should be touch-ups to only the new hair growth, and the frequency between treatments should be at least six, and preferably eight or more weeks. In other words, applying hair straightener to already relaxed hair will greatly increase the risk of breakage.

Another possible error with hair straighteners is that all chemical relaxer has to be completely removed with warm water. Of assistance, some shampoos contain "color action" agents, which indicate by the color of the lather, if all the relaxer chemical has been effectively removed. Most importantly, a neutralizing formula, or stabilizer, is added next to the hair to lower the $\mathrm{pH}$. If the hair is not neutralized and its $\mathrm{pH}$ not restored to normal levels, the chemical will continue to further weaken the hair shaft. Until this neutralizing step is completed, the hair is extremely sensitive and so all pulling, tugging, and excessive combing of the hair should be avoided until this maneuver is completed.

Of note, the use of hot styling tools including blow dryers, hot combs, and curling irons should be avoided, if possible, to hair that is relaxed. If one chooses to use the home relaxer box kits, it would be helpful to have someone else on hand to assist in the complete rinse and removal of the chemical.

Many stylists recommend applying a layer of petroleum jelly on the scalp before applying the relaxer to create a protective barrier between the chemical and the skin. Additionally, before using a relaxer, avoid scratching, brushing, and combing as this makes the scalp more susceptible to chemical irritation.

One must realize that the condition does not represent a failure of her hair to grow; but rather, an actual destruction of the hair follicle from the chemicals. It is hard to give up straight hair which you have grown comfortable with. Not that it helps, but you have been trying to make the hair different from what nature intended. By the way, the sodium hydroxide in relaxers is the same ingredient in drain cleaners, so it isn't unexpected that it causes hair breakage. The most practical therapy is avoidance of physical and chemical trauma to the hair. However, since many no longer yearn for the afro look, compromises may have to be considered. Some patients might merely have to use the products less frequently, or for less time duration. Many may be able to tolerate relaxers if they adhere to all of the safety precautions outlined above.

\section{Information on Telogen Effluvium}

Hair on the scalp typically grows for three to six years before going into a resting state for three months. This hair cycling is the reason why one notices a few hairs coming out with brushing and washing throughout one's life. Normally, $90 \%$ of scalp hairs are in the growing state. Average hair loss per day is twenty-five hairs because of this cycling process. However, up to 100 hairs lost per day is still within normal limits. 
With telogen effluvium, more hairs are in the resting state rather than the growing state. Therefore, one notices more hair shedding than normal, and less total hair on the scalp. Various degrees of hair loss can occur with telogen effluvium.

The cause of the body to direct more hair to go into the resting state than normal has to do with energy conservation - the body typically has a certain amount of energy it uses for making the brain, muscles, heart, and hair work and grow. If the body senses some stress, it will redirect the energy away from hair growth and direct this energy to more important functions (like fighting infection, or making the heart work faster). Such stresses that cause telogen effluvium can be emotional (i.e. loss of a loved one, divorce), or physical (i.e. childbirth, surgery, extreme dieting, drug reaction, severe infection). If there is no obvious source of stress in a severe case of telogen effluvium, I will often suggest a blood test looking for any clues as to what might be causing the body to redistribute its energy away from hair growth. If there is a serious problem detected by the blood test, I will call you within 24 hours of receiving these results.

The normal technique to assess hair cycling abnormalities is the simple pull test. Hair is pulled with gentle traction to assess the amount of shedding and to examine the proximal ends of the hairs.

This condition is self-limited, meaning that with time and elimination of the body's stresses, the hair will regrow. Telogen effluvian can occur at any age. The treatment that I suggest is taking zinc and biotin (which are over-thecounter minerals and vitamins sold at drug stores and at health food stores). These products may be in certain vitamin products. I would prefer if you took at least $8 \mathrm{mg}$ of zinc/day and $30 \mathrm{mcg} /$ day of biotin. With these products and removal of body stresses, the body should readjust with new hair growth in two to four months.

Society makes a person with hair loss feel uncomfortable. Remember that this condition does not lead to baldness, it is not a medical disaster, and the hair will regrow (eventually). You have to lose $80 \%$ of your scalp hairs for someone to notice definite baldness when passing you in the mall. So God has given us a lot of hair so that mild hair loss will not be readily noticed in our society.

\section{Information About Pattern Alopecia in Women}

To all women, hair loss is devastating. There are many concerns and fears associated with alopecia (a general term meaning hair loss). Additionally, there are many agents that claim to grow hair, but almost all of these products are more hype than science. Of note, even medicine and research hasn't solved this type of hair loss.

My job is to see if the hair loss is due to some medical problem (of the ovaries or adrenal gland) as well as directing you to reasonable treatments available for your condition.

Pattern alopecia is a common hereditary condition in which there is central diffuse hair thinning. Women with pattern alopecia usually first notice a gradual thinning of their hair mostly on the top of their heads and their scalps become more visible. The condition can be caused by (1) too little estrogen, or (2) too much testosterone (often referred to as male hormones, even thought women have small amounts normally).

Pattern alopecia secondary to lower estrogen levels often occurs with menopause when the ovaries stop producing estrogen, or after female surgery, such as a tubal ligation (in which the blood supply to the ovaries is compromised (or greatly reduced), causing a diminishment of estrogen levels and basically early menopause). If you fit into this category, we have 5 options for treatment. The best option for growing hair is to (1) take prescription oral supplemental estrogen, but only if your primary care physician feels you are a candidate, as estrogen does have side effects. The alternative option is (2) topical, over-the-counter Rogaine for Women. A third option would be (3) natural estrogens sold over-the-counter at drug stores health food stores (if your primary care physician agrees). Another choice would be (4) topical ketoconazole cream or shampoo which recently has been shown to somewhat suppress androgen receptors. (5) Low dose spironolactone $25 \mathrm{mg}$ daily also has shown merit when used in consort with Rogaine for Women. (Note: some physicians may feel comfortable expanding possible treatments to include propecia and higher dosages of spironolactone, but I feel both these alternatives are risky.)

Note: These suggested treatments may retard further hair loss fairly well, but one should expect only minimal regrowth of lost hair. In some cases of advanced hair loss, one may have to consider wigs and/or hair transplantation.

Pattern alopecia secondary to an increase in male hormones (specifically testosterone and 5-dihydroxyepiandrosterone) is determined by laboratory tests. These tests are important to obtain if under the age of 45. (Note: if you are over 45 and without an increase in facial hair, these tests offer little help in our evaluation.) These tests allow an assessment of your ovarian and adrenal secretions of these two hormones. These tests are performed preferentially during one's period. (If you are no longer having periods, then you can take the test at any time.) (Inasmuch as estrogen values fluctuate so much, most physicians feel that estrogen levels do not aid in the assessment of pattern alopecia.) Note: Make sure that the lab also sends the results also to your primary care physician. If these blood tests reveal an abnormality, I will call you.....however, your primary care physician would determine what course to take in that scenario. If your tests are normal, then you would follow the discussion listed above in the paragraph discussing pattern alopecia due to low estrogen.

\section{Information on Alopecia Areata}

Alopecia areata is a common hair loss disorder accounting for $2 \%$ of new outpatient visits to dermatologists. Indeed, Humphrey Bogart and J. D. Rockefeller both had this condition. Patients present with sharply outlined, shiny bald patches on the scalp. These bald spots appear suddenly without any preceding event. The hairs in these areas are basically "on strike." Everything is in place to produce hair, but the hair-making machine is no longer working. It is believed that the body's white blood cells have made a protein (or antibody) that has attached to the hair roots. 
This protein is impairing hair from growing normally.

The natural history of alopecia areata has been well studied. The prognosis for the majority of people with alopecia areata is excellent. Most patients have a few shiny bald patches that persist for 6 months to two years, and then enjoy complete and permanent regrowth of the lost hair. It is only in the minority of people with alopecia areata in which the hairs do not return. In such patients the hair loss may be long standing and may continue to spread over much of the scalp.

There is no treatment that is $100 \%$ effective for alopecia areata. Recent articles suggest new topical immunomodulators (namely, elidil or protopic) may be very effective. These drugs are not readily absorbed through scalp skin and so a topical agent improving its skin penetration is helpful. We are presently using a topical retinoid (such as tazorac, retin-A, or differin) for application at night, with elidel or protopic applied twice daily to the areas of hair loss. It often takes two months to see improvement with this therapy. Other treatment options are tried in two months if necessary.

The hairs that regrow in the affected areas are occasionally white, and sometimes quite fine. The tendency is for the hair to regrow in this condition within several months. However, an occasional patient may have recurrences. Unfortunately, there is that rare patient with this condition who gets worse, experiences entire loss of scalp hair, and does not respond to any therapy.

In rare instances, the condition has been associated with other diseases. Citing from a textbook by Moschella, "It should be emphasized that, from a practical standpoint, patients with alopecia areata are generally in good health and ordinarily do not require special study. Extensive laboratory work-ups fail to reveal any unusual findings associated with alopecia areata." Thus, unless there are other signs of medical problems, I do not usually pursue investigational blood studies with this condition.

For more information, go to the web site of the National Alopecia Areata Foundation: (www.naaf.org).

\section{What You Should Know About Excess Facial Hair}

Hirsutism is defined as excessive growth of course, dark hair in areas where women normally only grow fine hair, such as above the lip, on the chin and sideburns. It can be hereditary or it could be secondary to an excess of any of the hormones called androgens (male hormones). All women do make some of these hormones, but when they are at levels seen in men, then that can be a problem. I will offer you a screening blood test to check the levels of these hormones. If possible, get the blood test during your period. Also, please make sure that the blood drawers know to also send the results to your other doctors. I will only call you if your tests are abnormal.
Your primary care physician or gynecologist are better able to assess if there might be a serious medical condition causing the hirsutism. For example, I do not do pelvic exams. Some of the diseases to consider are polycystic ovary syndrome, idiopathic hirsutism, Cushing's disease, and hormone-secreting tumors of the ovary or adrenal. Your primary care physician or gynecologist would also be the one to decide on whether any oral drugs are indicated for your hirsutism such as oral contraceptives, antiandrogens (such as spironolactone, finasteride, cyproterone acetate, metformin).

The decision to treat excess facial hair is a matter of personal choice. Our culture often determines how much hair is cosmetically acceptable and how important it is to treat. The psychological impact of hirsutism can range from annoying to severely disabling.

In terms of topical measures for your excess facial hair, no topical treatment is perfect, as you probably already know.

First, some people lighten (bleach) their hair so that the course hair is less noticeable. Shaving is actually the most common way of controlling facial hair, with over $50 \%$ of females choosing this method. Shaving does not change hair diameter, but stubbles can look large to some individuals.

Plucking and waxing can be used to remove hair, but can cause pigmentary changes and scarring.

Chemical depilatories are available but can cause skin irritation.

Electrolysis damages individual hair follicles with an electric current so that they do not grow back. This treatment can leave pigmentary changes in some individuals and can be expensive. One would have to seek a qualified electrologist for this procedure.

Laser hair removal has reports of success, but it is expensive and often requires return treatments, as hair removal is not usually permanent by this method. You would have to seek a physician who does this procedure on your own (as I do not make any specific recommendations for this cosmetic procedure). If considering lasers, you might be smart to read on the internet as to which laser would work best for your skin type (i.e. ruby, alexandrite, and/or neodymium-yttrium) and then find a physician who has that particular laser.

Vaniqa is a skin cream approved by the FDA for treatment of unwanted facial hair. It does not remove hair permanently, but seems to slow its growth. It appears to offer limited benefit for patients in my opinion.

\section{REFERENCES}

[1] Cash TF. The psychology of hair loss and its implications for patient care. Clin Dermatol 2001; 19: 161-66. 
[2] Burkhart CG, Burkhart CN. Trichorrhexis nodosa revisited. SkinMed 2007; 6: 57-8.

[3] Rushton DH. Nutritional factors and hair loss. Clin Exp Dermatol 2002; 27: 396-404.

[4] Burkhart CN, Burkhart CG. Use of topical immunomodulators with agents to increase absorption. J Drugs Dermatol 2002; 1: 122-123.

[5] Ellis CN, Brown MR, Voorhees JJ. Sulfasalazine for alopecia areata. J Am Acad Dermatol 2002; 46: 541-4.
[6] Bakar O, Gurbuz O. Is there a role for sulfasalazine in the treatment of alopecia areata? J Am Acad Dermatol 2007; 57: 703-6.

[7] Madani S, Shapiro J. Alopecia areata update. J Am Acad Dermatol 2000; 42: 549-66.

[8] Tiggemann M, Lewis C. Attitudes toward women's' body hair: relationship with disgust sensitivity. Psychol Women Quar 2004; 28: 381-7.

(c) Burkhart and Burkhart; Licensee Bentham Open.

This is an open access article distributed under the terms of the Creative Commons Attribution License (http://creativecommons.org/licenses/by/2.5/), which permits unrestrictive use, distribution, and reproduction in any medium, provided the original work is properly cited. 